\title{
Multiple translational temperature model and its shock structure solution
}

\author{
Kun $\mathrm{Xu}^{*}$ \\ Department of Mathematics, The Hong Kong University of Science and Technology, Kowloon, Hong Kong \\ Eswar Josyula ${ }^{\dagger}$ \\ U.S. Air Force Research Laboratory, Wright-Patterson Air Force Base, Ohio 45433-7913 \\ (Received 16 November 2004; revised manuscript received 21 March 2005; published 31 May 2005)
}

\begin{abstract}
Within a shock layer, the translational motion of the gas is more energetic in the direction perpendicular to the shock front than in the direction parallel to the shock. To predict this translational nonequilibrium, a generalized gas-kinetic BGK model is proposed. With the adaptation of a continuum gas distribution function truncated up to the Navier-Stokes order and a generalized particle collision time, this newly constructed kinetic model is used in the monatomic gas shock structure calculations, where two translational temperatures inside the shock layer are well captured.
\end{abstract}

DOI: 10.1103/PhysRevE.71.056308

PACS number(s): 47.20.Bp, 05.20.Dd, 02.70.-c

\section{INTRODUCTION}

The hypersonic rarefied gas flow is characterized by the large region of translation nonequilibrium. For the NavierStokes equations, the random translational energy is usually modeled by a single gas temperature. In order to capture the translational nonequilibrium and compare it with the direct simulation Monte Carlo (DSMC) solution, many continuum models have been proposed. More than 20 years ago, based on the molecular dynamical simulation, Holian et al. [1] qualitatively evaluated the different temperature profiles, from which Holian conjectured that the viscosity and heat conduction coefficients in the shock layer should depend only on the temperature perpendicular to the shock front, which is higher than that in other directions. Based on Holian's conjecture [2], an excellent improvement between continuum model and MD simulation of Salomons and Mareschal [3] was obtained. Based on the Boltzmann equation, Candler et al. have formulated hydrodynamic equations up to the Navier-Stokes order with translational nonequilibrium in the shock structure calculations [4]. Even with multitranslational temperatures, as observed by many researchers, the Navier-Stokes equations of the classical hydrodynamics are still incapable of accurately describing the nonequilibrium wave phenomena. For example, the Navier-Stokes solutions present a thin shock thickness in comparison with the experiment's measurement. In order to improve the Navier-Stokes solutions, much effort has been spent on the construction of higher-order hydrodynamic equations, such as the Burnett and regularized Grad's solutions [5-8]. However, the implementation of a multiple translational temperature model in the Burnett or extended hydrodynamical equations has not been done yet. In this paper, we develop a multitranslational temperature kinetic model and use the gas-kinetic scheme [9] to obtain its shock structure solution.

\footnotetext{
*Email address: (makxu@ust.hk)

†Email address: (eswar.josyula@wpafb.af.mil)
}

\section{A MULTIPLE TRANSLATIONAL TEMPERATURE MODEL}

Many kinetic models have been proposed for the capturing of multitranslational temperature effect. Holway [10] used an ellipsoid Maxwellian for the gas distribution. In the one-dimensional case, the multiple temperature distribution function stands as

$$
\begin{aligned}
g= & \rho\left(\frac{\lambda_{x}}{\pi}\right)^{1 / 2}\left(\frac{\lambda_{y}}{\pi}\right)^{1 / 2}\left(\frac{\lambda_{z}}{\pi}\right)^{1 / 2} \exp \left[-\lambda_{x}(u-U)^{2}\right. \\
& \left.-\lambda_{y} v^{2}-\lambda_{z} w^{2}\right],
\end{aligned}
$$

where $\rho$ is the density, $U$ is the macroscopic velocity in the $x$ direction, and $(u, v, w)$ are the components of particle velocities in the $x, y$, and $z$ directions. The parameters $\lambda$ is related to the gas temperature, i.e., $\lambda_{x}=m / 2 k T_{x}, \lambda_{y}=m / 2 k T_{y}$, and $\lambda_{z}=m / 2 k T_{z}$. For the 1D shock structure, we have $T_{y}=T_{z}$; therefore, $\lambda_{y}=\lambda_{z}$. The establishment of the above ellipsoid Maxwell distribution is due to the course of particle collisions. Physically, the above ellipsoid equilibrium state $g$ will further approach an overall equilibrium $\bar{g}$ during the course of particle collisions

$$
\bar{g}=\rho\left(\frac{\lambda^{e q}}{\pi}\right)^{3 / 2} \exp \left\{-\lambda^{e q}\left[(u-U)^{2}+v^{2}+w^{2}\right]\right\} .
$$

With the mass, momentum, and total energy conservation during the particle collision, the relation between the absolute local equilibrium temperature $T^{e q}\left(\lambda^{e q}=m / 2 k T^{e q}\right)$ and the individual temperatures $T_{x}, T_{y}$, and $T_{z}$ is

$$
T^{e q}=\frac{1}{3}\left(T_{x}+2 T_{y}\right),
$$

where the assumption $T_{y}=T_{z}$ has been used in the current 1D case. The above process from $g$ to $\bar{g}$ can be modeled as a collision process with relaxation time $\tau$, i.e., $(\bar{g}-g) / \tau$. Taking moment $v^{2} / 2$ on the collision term $(\bar{g}-g) / \tau$ and using the relation (3), we can get the source term for the temperature evolution equation in the $y$ direction, which is $\rho R\left(T_{x}\right.$ $\left.-T_{y}\right) / 3 \tau$, where $R$ is the gas constant. This is exactly the 

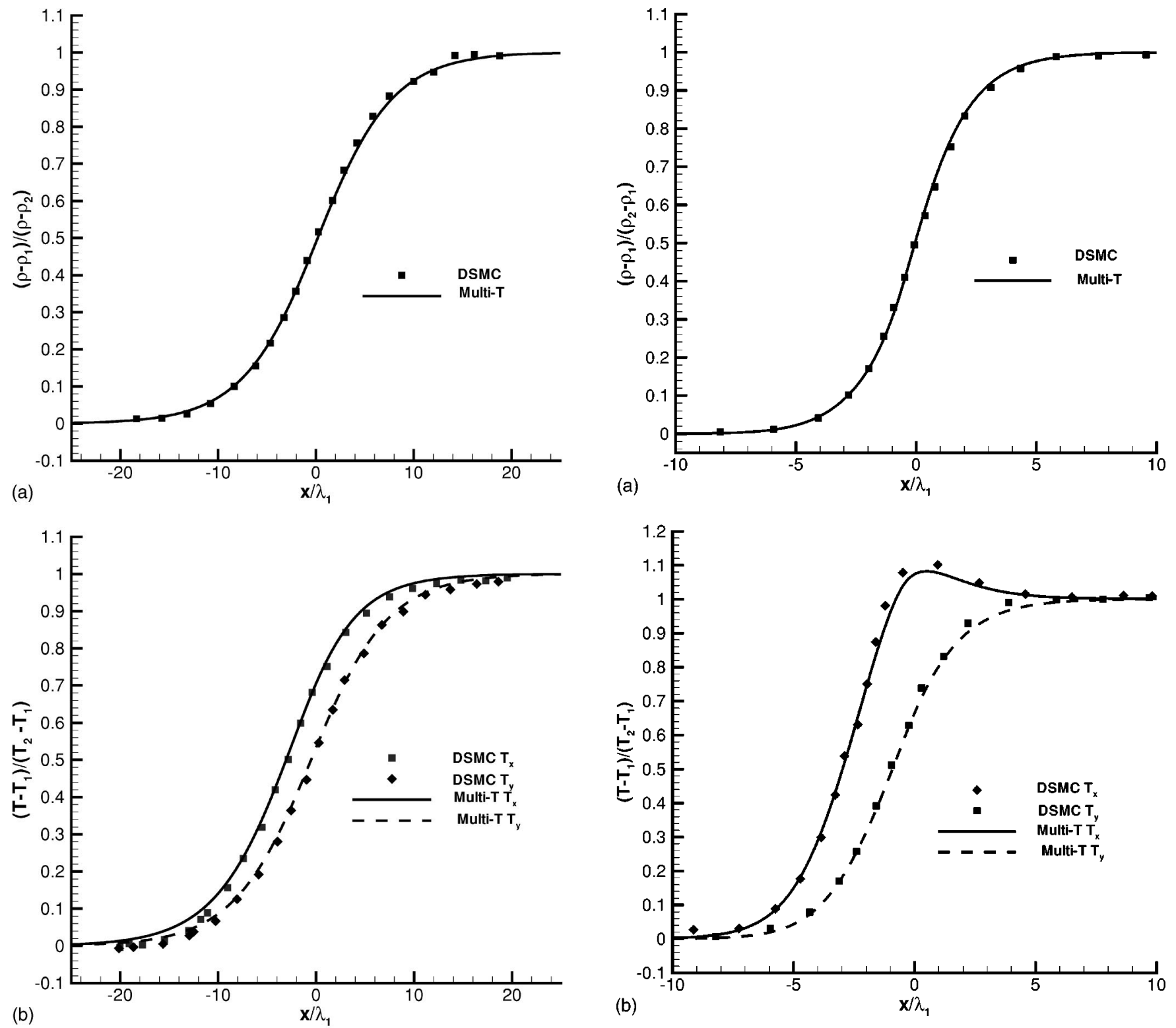

FIG. 1. Computed normalized density and temperatures for a Mach 1.2 Maxwellian gas shock wave. The DSMC solution is from Candler et al. [4].

same source term constructed by Candler et al. [4] in their extended Navier-Stokes equations based on the Boltzmann equation.

Starting a nonequilibrium gas distribution function $f$ for a monatomic gas, the particle collision will drive $f$ to $g$ and $\bar{g}$. It is hard to distinguish the process from $f$ to $g$ or from $f$ to $\bar{g}$. In terms of the particle collision time, they should be the same for the translational nonequilibrium. Therefore, for the monatomic gas we can construct a generalized BGK model for the translational nonequilibrium

$$
f_{t}+u f_{x}=\frac{1}{2}\left[\frac{g-f}{\tau}+\frac{\bar{g}-f}{\tau}\right],
$$

where $f$ is the real gas distribution function, and $g$ and $\bar{g}$ are the corresponding Maxwellians defined above. The use of

FIG. 2. Computed normalized density and temperatures for a Mach 2.0 Maxwellian gas shock wave.

the weight function $1 / 2$ in the above model is due to the following consideration. For any kinetic model for capturing multiple translational temperature, one basic requirement is that the averaged temperature, such as $T=\frac{1}{3}\left(T_{x}+2 T_{y}\right)$, should go back to the same temperature as that in the singletemperature BGK model [11]. Taking moments $(u-U)^{2}, v^{2}$ and $w^{2}$ on Eq. (4), we can obtain the temperature evolution equations in individual directions. Putting them together and using the condition $3 T=T_{x}+T_{y}+T_{z}$, we can also get the evolution equation for the averaged temperature $T$

$$
(\rho T)_{t}+(\rho U T)_{x}=\left(\rho T^{e q}-\rho T\right) / \tau .
$$

This is the same temperature evolution equation from the original BGK model. Therefore, the model (4) with a simple weight function is a natural extension from one to multiple temperatures. 

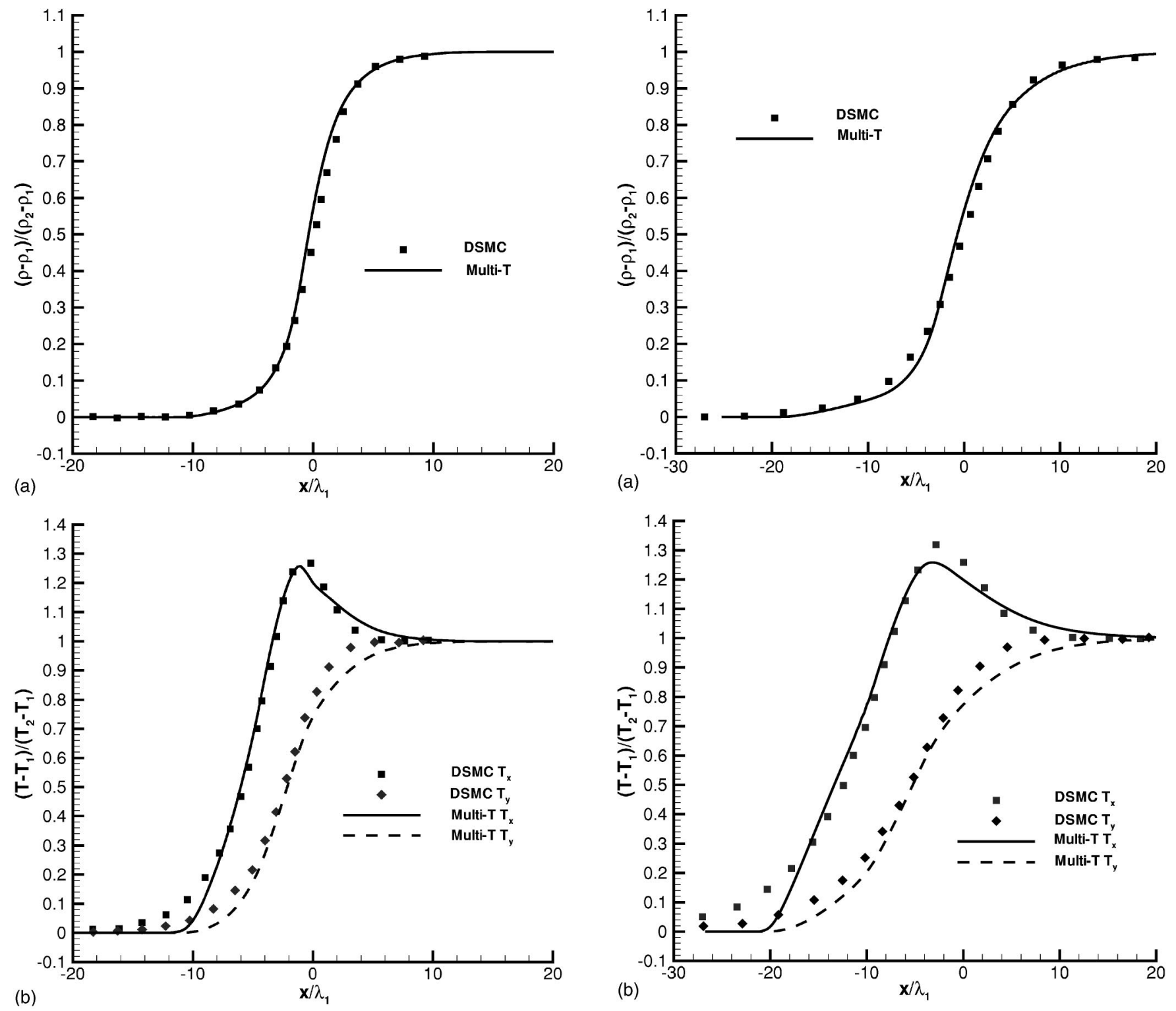

FIG. 3. Computed normalized density and temperatures for a Mach 5.0 Maxwellian gas shock wave.

The mass $\rho$, momentum $\rho U$, total energy $\rho E$, and thermal energy $\rho E_{r}$ in the $y$ - and $z$ directions are the moments of $f$

$$
W=\left(\begin{array}{c}
\rho \\
\rho U \\
\rho E \\
\rho E_{r}
\end{array}\right)=\int \psi f d u d v d w
$$

where $\psi$ has the components

$$
\boldsymbol{\psi}=\left(1, u, \frac{1}{2}\left(u^{2}+v^{2}+w^{2}\right), \frac{1}{2}\left(v^{2}+w^{2}\right)\right)^{T} .
$$

Since only mass, momentum, and total energy are conserved during particle collisions, the collision term in Eq. (4) satisfies the condition

FIG. 4. Computed normalized density and temperatures for a Mach 11 Maxwellian gas shock wave.

$$
\begin{gathered}
\int \frac{1}{2}[(g-f)+(\bar{g}-f)] \psi_{\alpha} d u d v d w=S=(0,0,0, s)^{T}, \\
\alpha=1,2,3,4,
\end{gathered}
$$

where the source term $s$ is modeled as $s=\rho R\left(T_{x}-T_{y}\right) / 3 \tau$.

In order to validate the above model, the numerical method developed in Ref. [9] is used to solve Eq. (4). This is a finite volume method

$$
W_{j}^{n+1}=W_{j}^{n}+\frac{1}{\Delta x} \int_{0}^{\Delta t}\left[F_{j-1 / 2}(t)-F_{j+1 / 2}(t)\right] d t+S_{j}^{n} \Delta t,
$$

where $W_{j}^{n}$ is the cell-averaged mass, momentum, total energy, and the thermal energy in the $y$ - and $z$ directions, and $F_{j+1 / 2}$ is the corresponding fluxes at a cell interface by solving Eq. (4). Note that $\Delta t$ is the time step $\Delta t=t^{n+1}-t^{n}$, and $S_{j}^{n}$ is the source term in the thermal energy equation. The evalu- 
ation of the fluxes is based on the gas distribution function $f$ at a cell interface. In the shock structure calculation, the gas distribution function at a cell interface is constructed using an iterative expansion of model (4), and has the form

$$
f=\frac{1}{2}(g+\bar{g})-\frac{1}{2} \tau\left[g_{t}+\bar{g}_{t}+u\left(g_{x}+\bar{g}_{x}\right)\right]+\frac{1}{2} t\left(g_{t}+\bar{g}_{t}\right),
$$

where the $g_{x}$ and $\bar{g}_{x}$ can be obtained from the gradients of macroscopic variables, and $g_{t}$ and $\bar{g}_{t}$ are evaluated based on the compatibility conditions

$$
\int\left(g_{t}+u g_{x}\right) \psi_{\alpha} d u d v d w=0 \text { and } \int\left(\bar{g}_{t}+u \bar{g}_{x}\right) \psi_{\alpha} d u d v d w=0 \text {. }
$$

The third term on the right-hand side of Eq. (7) is related to the time evolution part $f_{t}$, which is approximated by the time derivative of the equilibrium state $(g+\bar{g}) / 2$. The iterative method for the BGK solution has been proved to be equivalent to the Chapman-Enskog expansion up to the superBurnett order [12].

Even with the capacity of recovering two translational temperature, the nonequilibrium distribution (7) truncated up to the Navier-Stokes order is inadequate in the description of translational nonequilibrium. For example, the shock thickness constructed from the above model will be too thin in comparison with experimental measurements. In an earlier paper [13], we replace the collision time $\tau$ in the ChapmanEnskog expansion by a generalized one $\tau_{*}$, which is obtained by imposing a closed solution of the BGK model. In our current approach, a generalized particle collision time $\tau_{*}$ in Eq. (7) will also be used. The value of $\tau_{*}$ is obtained by

$$
\tau_{*}=\frac{\tau}{1+\tau\left\langle D^{2} g\right\rangle /\langle D g\rangle},
$$

where $D=\partial_{t}+u \partial_{x}$ and $\langle\cdots\rangle=\int(\ldots)(u-U)^{2} d u d v d w$; see Ref. [13]. Here, $\tau$ depends on the macroscopic variables through the relation $\tau p=\mu_{\text {ref }}\left(T / T_{\text {ref }}\right)^{\omega}$, and the generalized $\tau^{*}$ will depend not only on the macroscopic variables but also their gradients. In order to simulate the flow with any realistic Prandtl number, a modification of the heat flux in the energy transport at a cell interface, such as that used in Ref. [9], is also implemented in the current calculation.

\section{SHOCK STRUCTURE CALCULATION AND CONCLUSION}

One of the direct tests for the above model is the calculation of the shock structure. Since there are almost no experimental data for the two translational temperature measurements, the comparison with the DSMC and MD solution becomes necessary. The current calculation is for a monatomic gas with the atomic weight of argon and a Maxwellian (inverse fourth power) interatomic potential $\left(\mu_{\text {ref }}\right.$ $=2.515 \times 10^{-5} \mathrm{~kg} / \mathrm{ms}, T_{r e f}=273 \mathrm{~K}$, and $\omega=1$ for Maxwellian gas and $\omega=0.5$ for hard sphere). All computations use an equally spaced mesh with total 200 grid points with incoming gas condition $p_{1}=1171.88 \mathrm{~Pa}, T_{1}=226.64 \mathrm{k}$. Candler et al. [4] have extensively studied the shock structure with mul-

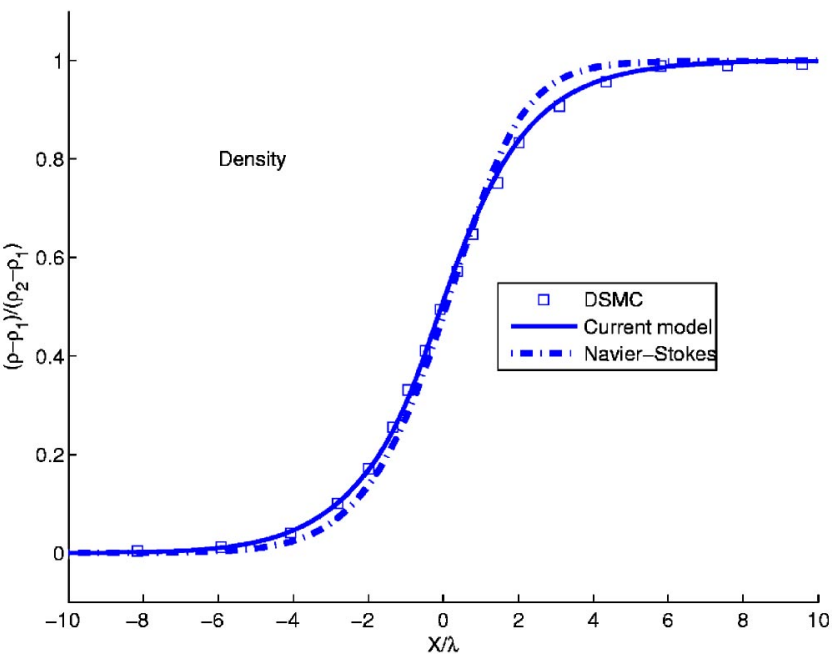

FIG. 5. Density distributions from NS, current model, and DSMC for a Mach 2.0 Maxwellian gas.

tiple translational temperature; their DSMC results will be used here as a benchmark solution.

Figures 1-4 plot the Maxwellian gas shock waves for free-stream Mach numbers between 1.2 and 11. We plot the normalized temperature, $T_{n}=\left(T-T_{1}\right) /\left(T_{2}-T_{1}\right)$, and normalized density $\rho_{n}=\left(\rho-\rho_{1}\right) /\left(\rho_{2}-\rho_{1}\right)$ versus $x / \lambda_{1}$. The upstream mean-free paths $\lambda_{1}$ as defined in Ref. [4] have the value $\lambda_{1}$ $=2 \mu / \rho_{1} \bar{C}_{1}$, where $\bar{C}_{1}$ is the mean atomic speed at the upstream condition. From these figures, we can see the reasonable agreement between the current results and DSMC solutions for all cases from the continuum $(M=1.2)$ to the highly nonequilibrium one $(M=11)$. We also calculate the asymmetry factor $\left(Q_{\rho}\right)$ for the $M=2$ case, which is defined by $Q_{\rho}$ $=\int_{-\infty}^{x^{*}} \rho_{n} d x / \int_{x^{*}}^{\infty}\left(1-\rho_{n}\right) d x$, where $x^{*}$ is the location for $\rho_{n}=0.5$. Figure 5 shows the density distributions from the current solution, Navier-Stokes [9], and DSMC solutions. The calculated $Q_{\rho}$ 's from the current model and the Navier-Stokes are
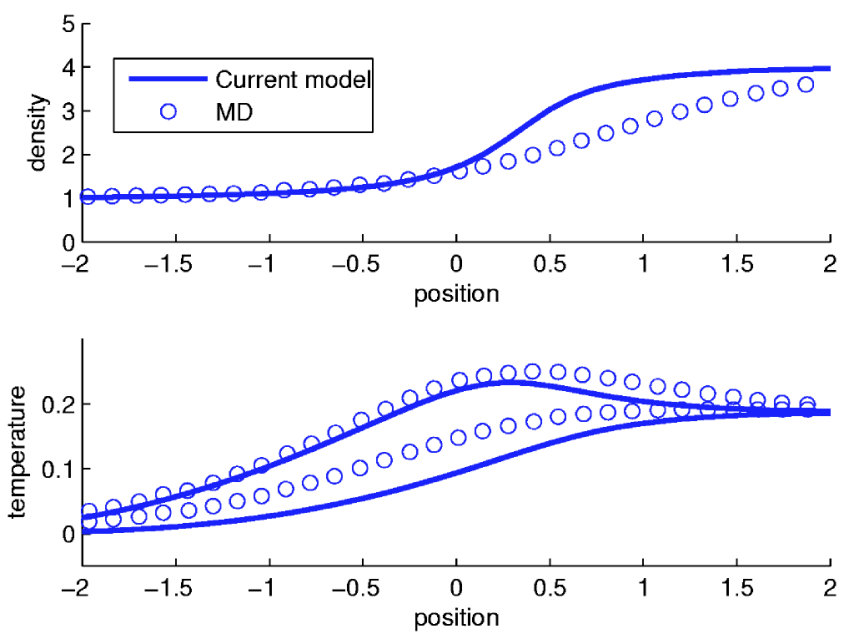

FIG. 6. Density $\rho / \rho_{1}$ (up) and temperature $T /\left(U_{1}^{2} / R\right)$ (down) distributions vs position $x / l$ in units of upstream mean-free path $(l)$ for an ideal hard sphere gas at $M=134$ : solid lines (current model) and circles (MD solution) in Ref. [2]. 
0.9239 and 1.251 , respectively. Last, we apply the current model to the high Mach number $M=134$ shock wave for hard sphere gas with the same incoming flow condition. The density and temperature distributions are shown in Fig. 6, where the MD solution is from Ref. [2]. At such a high Mach number, the downstream temperature goes up to 1.273 million degrees. The physical validity for any model presented in this paper at such an extreme condition may be questionable.

In this paper, we construct a generalized BGK model to capture the multiple translational temperature for a monatomic gas. The current model is a natural extension of the single-temperature BGK model. The averaged temperature in the current model will go back to the single temperature in the original BGK equation. Since continuous particle velocity space is used in the current numerical approach, the efficiency of the current method is close to the direct NavierStokes flow solver, which is much more efficient than the direct Boltzmann and DSMC methods. All shock structures in this paper are obtained numerically based on the kinetic model.

\section{ACKNOWLEDGMENTS}

This research was supported by Hong Kong Research Grant Council HKUST6116/03E and 6102/04E, by the U.S. Air Force Research Laboratory, and by the U.S. Air Force Office of Scientific Research.
[1] B. L. Holian, W. G. Hoover, B. Moran, and G. K. Straub, Phys. Rev. A 22, 2798 (1980).

[2] B. L. Holian, C. W. Patterson, M. Mareschal, and E. Salomons, Phys. Rev. E 47, R24 (1993).

[3] E. Salomons and M. Mareschal, Phys. Rev. Lett. 69, 269 (1992).

[4] G. V. Candler, S. Nijhawan, D. Bose, and I. D. Boyd, Phys. Fluids 6, 3776 (1994).

[5] X. Zhong, R. W. MacCormack, and D. R. Chapman, AIAA J. 31, 1036 (1993).

[6] F. J. Uribe, R. M. Velasco, and L. S. Garcia-Colin, Phys. Rev.
E 58, 3209 (1998).

[7] R. K. Agarwal, K. Y. Yun, and R. Balakrishnan, Phys. Fluids 13, 3061 (2001).

[8] R. Balakrishnan, J. Fluid Mech. 503, 201 (2004).

[9] K. Xu, J. Comput. Phys. 171, 289 (2001).

[10] L. H. Holway, JR., Phys. Fluids 9, 1658 (1966).

[11] P. L. Bhatnagar, E. P. Gross, and M. Krook, Phys. Rev. 94, 511 (1954).

[12] T. Ohwada and K. Xu, J. Comput. Phys. 201, 315 (2004).

[13] K. Xu, Phys. Fluids 14, L17 (2002). 